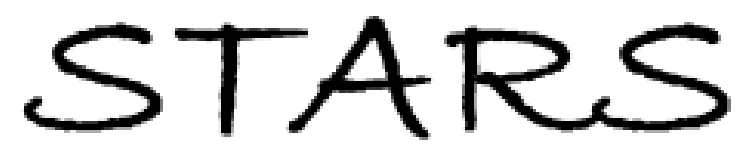

University of Central Florida

STARS

$1-1-2008$

\title{
Thermal resistivity of Si-Ge alloys by molecular-dynamics simulation
}

\author{
Ashton Skye \\ University of Central Florida \\ Patrick K. Schelling \\ University of Central Florida
}

Find similar works at: https://stars.library.ucf.edu/facultybib2000 University of Central Florida Libraries http://library.ucf.edu

This Article is brought to you for free and open access by the Faculty Bibliography at STARS. It has been accepted for inclusion in Faculty Bibliography 2000 s by an authorized administrator of STARS. For more information, please contactSTARS@ucf.edu.

\section{Recommended Citation}

Skye, Ashton and Schelling, Patrick K., "Thermal resistivity of Si-Ge alloys by molecular-dynamics simulation" (2008). Faculty Bibliography 2000s. 997.

https://stars.library.ucf.edu/facultybib2000/997

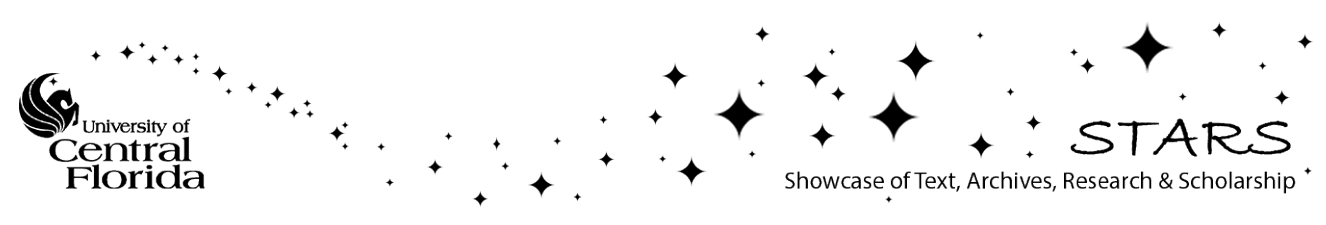




\section{Thermal resistivity of Si-Ge alloys by molecular-dynamics simulation}

Cite as: J. Appl. Phys. 103, 113524 (2008); https://doi.org/10.1063/1.2936868

Submitted: 13 November 2007 . Accepted: 25 March 2008 . Published Online: 13 June 2008

Ashton Skye, and Patrick K. Schelling

\section{ARTICLES YOU MAY BE INTERESTED IN}

Phonon transport analysis of silicon germanium alloys using molecular dynamics simulations Journal of Applied Physics 113, 203514 (2013); https://doi.org/10.1063/1.4807301

Thermal conductivity of Si-Ge superlattices

Applied Physics Letters 70, 2957 (1997); https://doi.org/10.1063/1.118755

Intrinsic lattice thermal conductivity of semiconductors from first principles

Applied Physics Letters 91, 231922 (2007); https://doi.org/10.1063/1.2822891

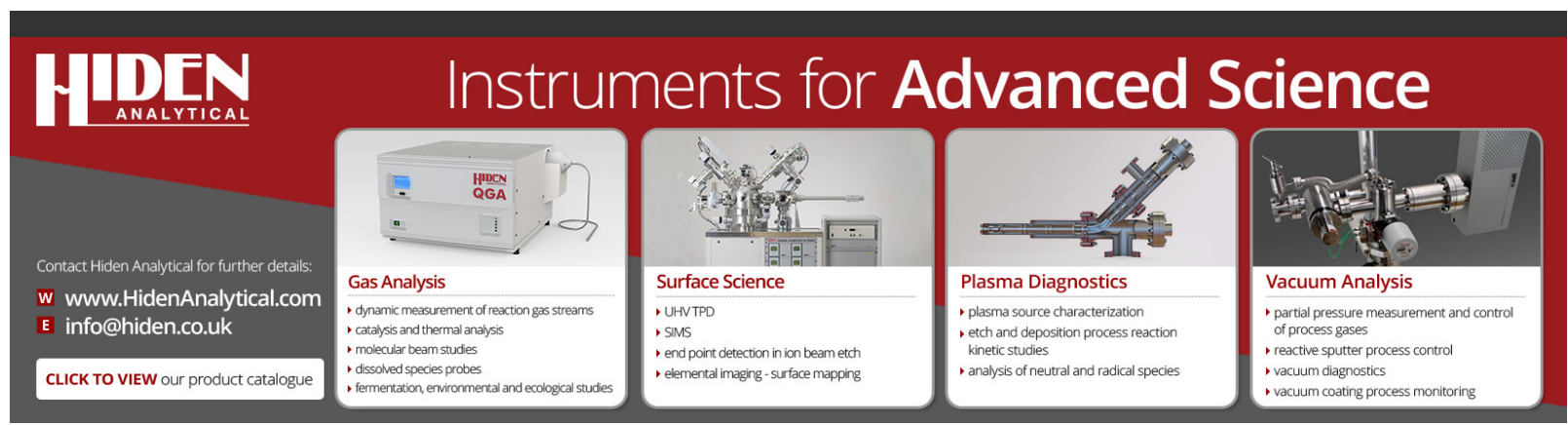




\title{
Thermal resistivity of Si-Ge alloys by molecular-dynamics simulation
}

\author{
Ashton Skye and Patrick K. Schelling ${ }^{\text {a) }}$ \\ Advanced Materials Processing and Analysis Center and Department of Physics, \\ University of Central Florida, Orlando, Florida 32816, USA
}

(Received 13 November 2007; accepted 25 March 2008; published online 13 June 2008)

\begin{abstract}
We explore the ability of molecular-dynamics simulation to elucidate thermal transport in $\mathrm{Si}-\mathrm{Ge}$ alloys. Simulations using Stillinger-Weber potentials yield values for the thermal resistivity significantly higher than experimental measurements. In agreement with experiment and theoretical predictions, we find that scattering from mass disorder is dominant, with bond disorder and strain effects playing a very minor role. To explore the origins of the large discrepancies with experiment, we use theoretical methods suitable for the limit where point-defect scattering dominates the resistivity. We find that point-defect scattering models based on a Debye spectrum cannot be used to fit our simulations, indicating that high-frequency modes may play an important role in the simulation. The results have important implications for using classical molecular-dynamics simulation to predict properties of alloy materials near and below the Debye temperature.
\end{abstract}

(C) 2008 American Institute of Physics. [DOI: 10.1063/1.2936868]

\section{INTRODUCTION}

Silicon-germanium alloys are of interest for several applications, including high-mobility transistors ${ }^{1}$ and thermoelectrics. ${ }^{2,3}$ In any application, the low thermal conductivity observed in $\mathrm{Si}-\mathrm{Ge}$ alloys is an important consideration. For example, in thermoelectric applications, the low thermal conductivity of the alloy tends to result in higher thermoelectric efficiency. Atomic-level simulation has not yet played a significant role in elucidating the properties of bulk $\mathrm{Si}-\mathrm{Ge}$ alloys or nanostructures made from $\mathrm{Si}$ and $\mathrm{Ge}$ and their alloys. It is very likely that advances in thermoelectrics will depend on some combination of alloy scattering with other scattering mechanisms. ${ }^{4}$ In the case of Si-Ge materials, significant promise exists for nanowire materials which combine alloy scattering, interface scattering, and confinement effects. ${ }^{5}$ Recent experimental work has shown that embedding nanoparticles in an alloy is another promising approach that can result in low thermal conductivity and high thermoelectric efficiency. ${ }^{6}$ Atomic-scale simulations can play an important role in elucidating scattering mechanisms for promising nanomaterials comprised partly of alloys. However, it is crucial to first establish the ability of simulation to predict transport properties of alloys.

The Si-Ge alloys are an ideal model system to explore the potential and limitations of atomic-scale simulation. Both $\mathrm{Si}$ and Ge have a ground-state diamond-lattice structure. The room-temperature lattice parameters of $\mathrm{Si}$ and $\mathrm{Ge}$ are 5.43 and $5.66 \AA$, respectively. The structure has been shown experimentally and by simulation ${ }^{7}$ to be truly random, with no appreciable long- or short-range compositional ordering. Theoretical predictions suggest that phonon scattering in $\mathrm{Si}-\mathrm{Ge}$ alloys mostly results from mass disorder, and bond disorder plays a relatively minor role. ${ }^{8}$ This suggests that even a simple model which accounts for the mass disorder

\footnotetext{
a) Author to whom correspondence should be addressed. Electronic mail: pschell@mail.ucf.edu.
}

might be adequate to make predictions. In short, because the scattering mechanisms are simple and the structure is well understood, it should be possible to make direct comparisons between experiment and simulation.

In this paper, we report an extensive molecular-dynamics (MD) study of the thermal resisitivity of bulk $\mathrm{Si}-\mathrm{Ge}$ alloys as a function of temperature and composition. Our results are compared to experiment and theoretical models. By using MD simulation, we are able to differentiate the effects of mass disorder and bond disorder. In particular, we report results for thermal conductivity in an alloy where the atomic mass is varied while keeping the interactions uniform. In agreement with theoretical predictions, we find that the mass differences play a significantly larger role in phonon scattering than the differences in the chemical bonds introduced by alloying. However, we find that the simulations greatly exaggerate the scattering due to point defects. We discuss some of the possible reasons for the significant disagreement between experiment and simulation.

In the next section, we briefly describe the details of the simulations. In Sec. III, the results are presented, followed in Sec. IV by a theoretical analysis and comparison to previous works. Finally, in Sec. V we discuss the implications of this study.

\section{SIMULATION METHOD}

The Stillinger-Weber (SW) potential uses two-body and three-body terms to stabilize the diamond lattice. The original parameters were developed to provide an approximate description of condensed phases of $\mathrm{Si}^{9}{ }^{9}$ Parameters for $\mathrm{Ge}$ were determined by fitting to experimental data. ${ }^{10}$ In the simulation studies described here, we adopt the parameters from Refs. 9 and 10 to describe $\mathrm{Si}-\mathrm{Si}$ and $\mathrm{Ge}-\mathrm{Ge}$ interactions. To treat $\mathrm{Si}-\mathrm{Ge}$ interactions, we follow previous authors and use the geometric mean of the interaction parameters for the pure substances. ${ }^{7,11}$ Using this model, it has been shown 


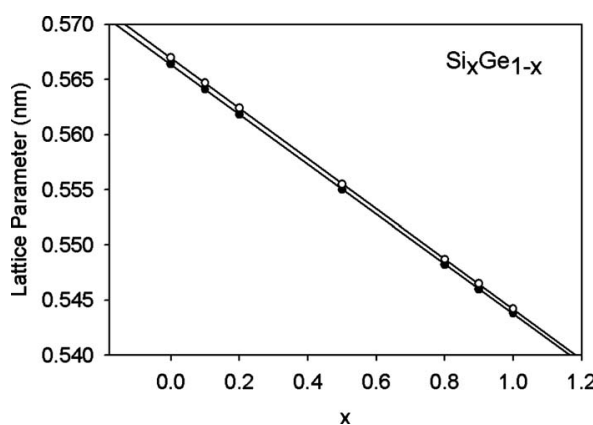

FIG. 1. Computed lattice parameters of $\mathrm{Si}_{x} \mathrm{Ge}_{1-x}$ as a function of composition $x$ for $T=300 \mathrm{~K}$ (closed circles) and $T=500 \mathrm{~K}$ (open circles).

previously using Monte-Carlo simulation that the bulk alloy exhibits no compositional order, in agreement with experiment. $^{7}$

We first computed the time-averaged lattice parameters in the constant pressure and temperature ensemble using the Parrinello-Rahman algorithm ${ }^{12}$ at zero external pressure. We used a supercell with dimensions of $8 \times 8 \times 8$ nonprimitive unit cells and a total of 4096 atoms. Each composition was simulated for $27.6 \mathrm{ps}$ until equilibrated, followed by $55.2 \mathrm{ps}$ of simulation to obtain converged averages. The results are shown in Fig. 1 for $\mathrm{Si}_{x} \mathrm{Ge}_{1-x}$ as a function of the composition parameter $x$ at $T=300$ and $500 \mathrm{~K}$. The results in Fig. 1 agree very well with previous MD studies using the SW potential of $\mathrm{Si}^{13} \mathrm{Ge},{ }^{11}$ and bulk $\mathrm{Si}-\mathrm{Ge}$ alloys. ${ }^{11}$ In particular, the lattice parameter of $\mathrm{Si}_{x} \mathrm{Ge}_{1-x}$ follows Vergard's law quite closely, which is a linear interpolation between the lattice constants $a_{\mathrm{Si}}$ for silicon and $a_{\mathrm{Ge}}$ for germanium,

$$
a(x)=x a_{\mathrm{Si}}+(1-x) a_{\mathrm{Ge}} .
$$

Nonequilibrium MD simulation based on the approach developed by Jund and Jullien was used to compute the thermal conductivity. ${ }^{14,15}$ In this approach, a thin region of the system is heated by rescaling atomic velocities. Far from the heated region, atomic velocities are rescaled to result in cooling. At each step, the amount of energy added to the heated region is equal to the amount of energy removed from the cooled region. As a result, the algorithm conserves energy, even though the atomic dynamics in the heated and cooled regions is drastically altered. The resulting temperature gradient is computed, and then Fourier's law is used to determine the thermal conductivity from $J=-\kappa(\partial T / \partial z)$, where $J$ is the thermal current and the $z$-axis is coincident with the long axis of the simulation cell. We show a typical temperature profile in Fig. 2 for $x=0.80$ and an average temperature $T=300 \mathrm{~K}$.

The simulation supercell for the results reported in the next section had dimensions of $4 \times 4 \times 128$ nonprimitive unit cells with a total of 16384 atoms. Periodic boundary conditions were applied in all three dimensions. For pure $\mathrm{Si}$, the length of the simulation cell $L_{z}$ is about $69.5 \mathrm{~nm}$. For pure $\mathrm{Ge}$ the lattice parameter is slightly larger, and the length $L_{z}$ of the simulation cell was about $72.4 \mathrm{~nm}$. For the alloys, the dimensions of the supercell were determined from the data shown in Fig. 1. In each case, thermal conductivity calculations were performed using a system with a fixed volume. The regions where heating and cooling occur were taken to

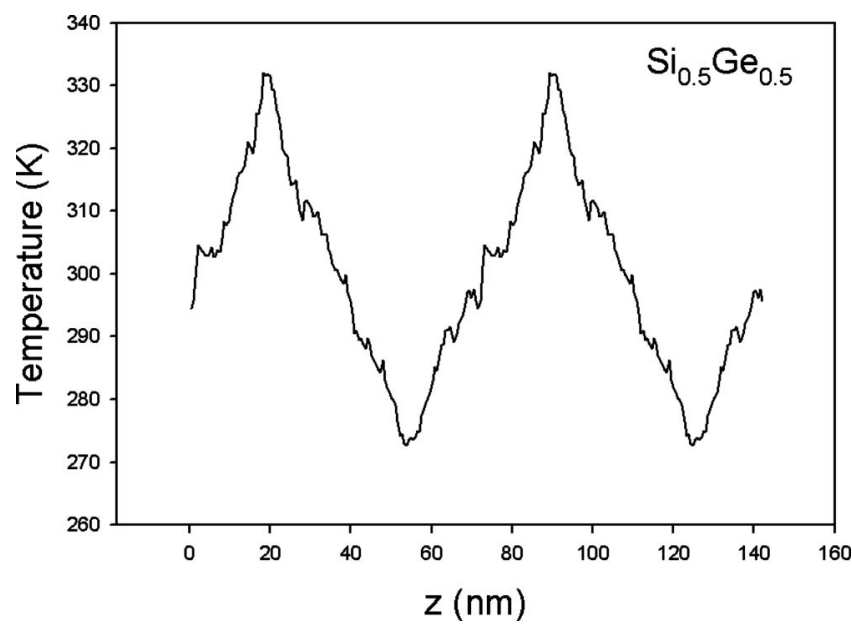

FIG. 2. Simulation temperature profile for $x=0.50$ at $T=300 \mathrm{~K}$. The fit temperature profile for this data results in a conductivity of $\kappa$ $=1.06 \mathrm{~W} / \mathrm{mK}$.

be in each case six nonprimitive cells in length. The system was first equilibrated at constant temperature for 110 ps at the desired temperature. After the initial equilibration, the heat source and sink were switched on. Except where noted in the next section, we include 1.12 ns of simulation with the heat source and sink on. However, we found that about 0.56 ns of simulation time was required in order to reach a steadystate temperature gradient. Therefore, the temperature profile in Fig. 2 and those used to compute $\kappa$ in the next section were averaged only over the final $0.56 \mathrm{~ns}$ of the simulation. The heat current in each alloy simulation was approximately $J \approx 1.4-1.5 \mathrm{GW} / \mathrm{m}^{2}$. While these heat currents are rather large, they are actually smaller than previous detailed studies in pure $\mathrm{Si}$ where the applicability of Fourier's law was verified. ${ }^{15}$ For pure Si systems, the value of $\kappa$ increases substantially, and the simulated heat current used in those cases was increased by a factor of 10 so that an appreciable thermal gradient could be accurately computed. For the numerical fitting used to obtain the thermal gradient, we exclude the regions of the hot and cold reservoirs, and also two additional nonprimitive cells $(\sim 1.1 \mathrm{~nm})$ on either side of the reservoirs. In contrast to our prior study of pure $\mathrm{Si}^{15}$ the temperature profiles for alloys are quite linear, even near the reservoir regions, as seen from the profile in Fig. 2. This linearity results from the strong defect scattering which limits the mean-free path of the heat carriers and restores local equilibrium very near to the hot and cold reservoirs. We find no dependence in our simulated values of $\kappa$ on the particular choice of initial random velocities.

\section{RESULTS}

We first computed the thermal conductivity of pure $\mathrm{Si}$ and $\mathrm{Ge}$ as a function of temperature. The results are shown in Table I for $T=300$ and $500 \mathrm{~K}$. For $\mathrm{Si}$, we found that our results are in good agreement with prior work. ${ }^{15}$ We have previously show that finite-size effects are important to take into account in order to compare to experimental values. ${ }^{15}$ Extrapolation to an infinite-size system yielded $\kappa_{\mathrm{Si}}$ $=119 \mathrm{~W} / \mathrm{mK}$ at $T=500 \mathrm{~K} .{ }^{15}$ For the current study, we have 
TABLE I. Simulated thermal conductivity $\kappa$ of pure $\mathrm{Si}$ and $\mathrm{Ge}$ at $T$ $=300 \mathrm{~K}$ and $T=500 \mathrm{~K}$. Finite-size effects cause the computed values to be substantially less than experimental measurements. This point is discussed in depth in Ref. 15

\begin{tabular}{ccc}
\hline \hline Material & $\kappa_{T=300 \mathrm{~K}}(\mathrm{~W} / \mathrm{mK})$ & $\kappa_{T=500 \mathrm{~K}}(\mathrm{~W} / \mathrm{mK})$ \\
\hline \multirow{2}{*}{$\mathrm{Si}$} & $27.0 \pm 2.7$ & $25.5 \pm 2.6$ \\
$\mathrm{Ge}$ & $19.0 \pm 1.9$ & $16.7 \pm 1.7$ \\
\hline
\end{tabular}

not extrapolated our results to an infinite size system for pure Ge or for pure $\mathrm{Si}$ at $T=300 \mathrm{~K}$. However, we can see that the values of $\kappa_{\mathrm{Ge}}$ and $\kappa_{\mathrm{Si}}$ are higher at $T=300 \mathrm{~K}$ than at $T$ $=500 \mathrm{~K}$. While the differences are fairly small, based on our prior results ${ }^{15}$ we expect that extrapolation to infinite size should yield the approximately linear dependence on temperature seen in the experiment. One problem in comparing the pure Ge results to experiment is that Ge has a large isotope concentration which is not simulated here. Because the focus of the present study is on alloy scattering, isotope scattering will play a very small role. Finally, the ratio $\kappa_{\mathrm{Si}}: \kappa_{\mathrm{Ge}}$ is 1.42 at $T=300 \mathrm{~K}$ and 1.53 at $T=500 \mathrm{~K}$. These are not too different from the ratio $\sqrt{\left(M_{\mathrm{Ge}} / M_{\mathrm{Si}}\right)}=1.61$, where $M_{\mathrm{Ge}}=72.61$ and $M_{\mathrm{Si}}=28.09$ are the atomic masses.

The simulated values of $\kappa$ for bulk alloys at $T=300$ and $500 \mathrm{~K}$ are shown in Table II. To facilitate comparison to theory in the next section, we plot, instead of the conductivity $\kappa$, the thermal resistivity $W=1 / \kappa$ as a function of composition for $T=300 \mathrm{~K}$ (Fig. 3) and $T=500 \mathrm{~K}$ (Fig. 4). We observe that the temperature dependence of $W$ cannot be resolved by our results. The estimated error in $\kappa$ of about $\pm 15 \%$ was determined by comparison to fits over several shorter segments of the total simulation time. This implies that the difference in $W$ between the two simulated temperatures is smaller than the numerical error inherent in our calculations. By contrast, experimental results show a substantial temperature dependence. In particular, it has been shown that experimental data for the resistivity $W$ from $T$ =300-900 K scales approximately as $T^{1 / 2}{ }^{8} \mathrm{We}$ conclude that the simulations are not accurately reproducing the experimental temperature dependence of $W$ for alloys. While longer simulations might be used to reduce numerical error and accurately determine the temperature dependence of $W$, we still expect the results to be dramatically different than experimental observation. We believe that the strong disagreement results from overly strong point-defect scattering in the simulations. This point will be demonstrated in Sec. IV.

TABLE II. Simulated thermal conductivity $\kappa$ of bulk alloys $\mathrm{Si}_{x} \mathrm{Ge}_{1-x}$ at $T$ $=300 \mathrm{~K}$ and $T=500 \mathrm{~K}$. The values for the pure systems correspond to $x$ $=0$ and $x=1$ and are given in Table $\mathrm{I}$. The temperature dependence is very weak for the alloys and cannot be established accurately using these results.

\begin{tabular}{ccc}
\hline \hline $\mathrm{X}$ & $\kappa_{T=300 \mathrm{~K}}(\mathrm{~W} / \mathrm{mK})$ & $\kappa_{T=500 \mathrm{~K}}(\mathrm{~W} / \mathrm{mK})$ \\
\hline 0.1 & $3.32 \pm 0.50$ & $3.50 \pm 0.53$ \\
0.2 & $2.09 \pm 0.31$ & $1.78 \pm 0.27$ \\
0.5 & $1.06 \pm 0.16$ & $1.14 \pm 0.17$ \\
0.8 & $1.39 \pm 0.21$ & $1.50 \pm 0.23$ \\
0.9 & $2.37 \pm 0.36$ & $2.69 \pm 0.40$ \\
\hline \hline
\end{tabular}

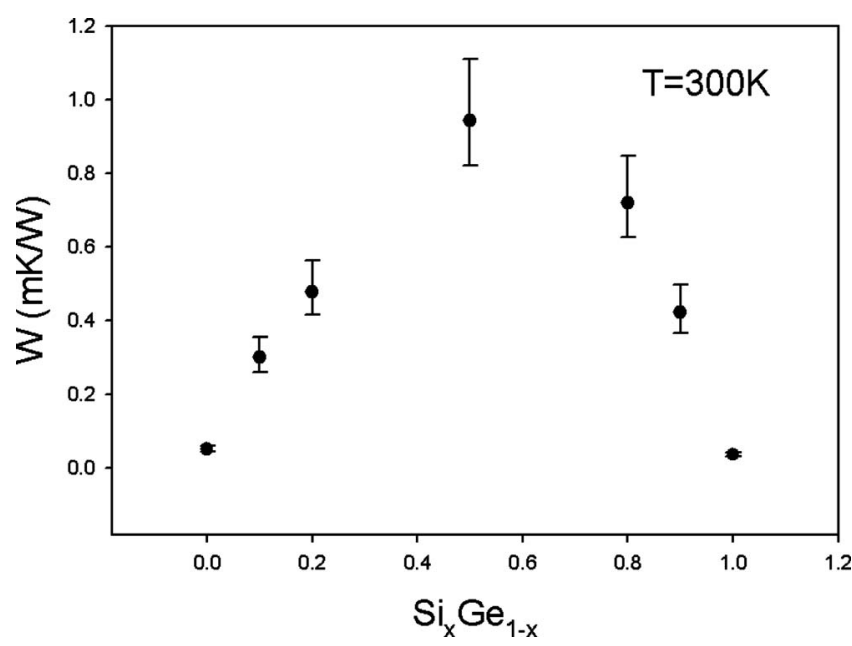

FIG. 3. Thermal resistivity $W$ for the alloys $\operatorname{Si}_{x} \mathrm{Ge}_{1-x}$ as a function of composition $x$ for $T=300 \mathrm{~K}$.

Theoretical predictions due to Abeles ${ }^{8}$ indicate that bond disorder and lattice strain due to alloying contribute very little to the thermal resistivity. In particular, theoretical models fit to experiment demonstrate that scattering from bond disorder and lattice strain explain only about $10 \%$ of the total resistivity $W$. The flexibility of MD simulations allows us to test this point directly. In Table III we present the thermal conductivities of pure and alloy systems with mass disorder and all interactions described by either the $\mathrm{Si}$ or the Ge potentials at $T=300 \mathrm{~K}$. The results in each case are quite close to those presented in Tables I and II and Fig. 3. This clearly demonstrates that the mass disorder plays a dominant role in scattering the lattice vibrations. The results also show that the Ge potential results in systematically lower $W$ than the $\mathrm{Si}$ potential. We believe that this arises from the tendency of SW potentials to provide a poor description of the dispersion of the transverse-acoustic (TA) phonon branch. It has previously been shown that the SW potential for Ge exaggerates the frequencies of the zone-edge TA modes more significantly than does the SW potential for Si. ${ }^{10,13}$

Finally, we have explored finite-size effects for the alloy with $x=0.80$ for $T=300 \mathrm{~K}$. We find that the thermal conduc-

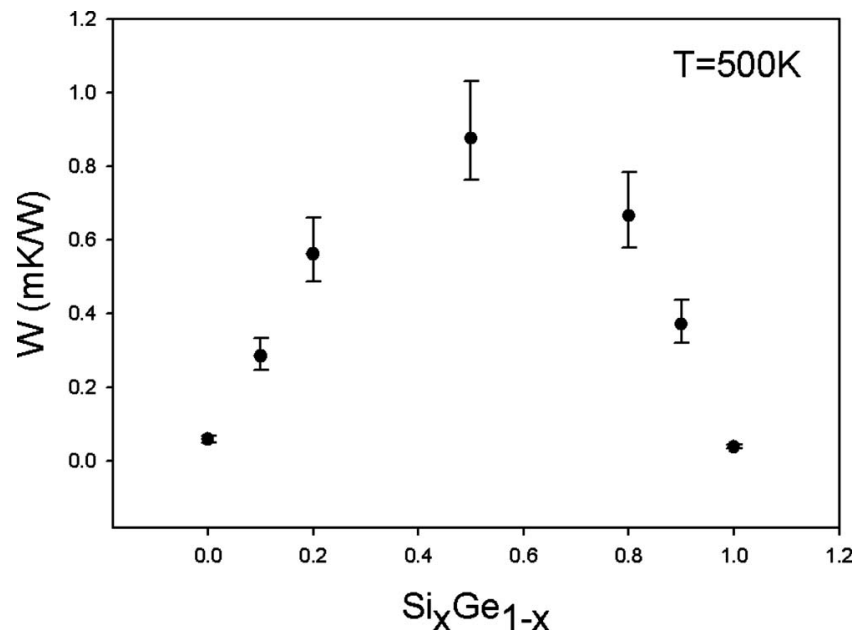

FIG. 4. Thermal resistivity $W$ for the alloys $\mathrm{Si}_{x} \mathrm{Ge}_{1-x}$ as a function of composition $x$ for $T=500 \mathrm{~K}$. 
TABLE III. Simulated thermal conductivity $\kappa$ of bulk alloys $\mathrm{Si}_{x} \mathrm{Ge}_{1-x}$ at $T$ $=300 \mathrm{~K}$ with only mass disorder. The results for $\kappa_{\mathrm{Si}}$ were obtained using the Si potential for all interactions, and the results for $\kappa_{\mathrm{Ge}}$ were obtained using only the Ge potential. The results show a trend for $\kappa_{\mathrm{Ge}}$ to be greater than $\kappa_{\mathrm{Si}}$.

\begin{tabular}{ccc}
\hline \hline $\mathrm{X}$ & $\kappa_{\mathrm{Si}}(\mathrm{W} / \mathrm{mK})$ & $\kappa_{\mathrm{Ge}}(\mathrm{W} / \mathrm{mK})$ \\
\hline 0.0 & $15.7 \pm 1.6$ & $19.0 \pm 1.9$ \\
0.1 & $2.77 \pm 0.42$ & $3.05 \pm 0.46$ \\
0.2 & $1.94 \pm 0.29$ & $2.09 \pm 0.31$ \\
0.5 & $1.08 \pm 0.16$ & $1.07 \pm 0.16$ \\
0.8 & $1.29 \pm 0.19$ & $1.43 \pm 0.21$ \\
0.9 & $2.06 \pm 0.31$ & $3.20 \pm 0.48$ \\
1.0 & $27.0 \pm 2.7$ & $26.4 \pm 2.6$ \\
\hline \hline
\end{tabular}

tivity increases somewhat as the system size increases. In particular, we obtain $\kappa=1.49 \mathrm{~W} / \mathrm{mK}$ for $L_{z}=140.3 \mathrm{~nm}$, and $\kappa=1.67 \mathrm{~W} / \mathrm{mK}$ for $L_{z}=210.5 \mathrm{~nm}$. While numerical error makes it hard to exactly determine the length dependence, extrapolation to $1 / L_{z}=0$ shows that $\kappa$ is at most $2.0 \mathrm{~W} / \mathrm{mK}$ for $x=0.80$ at $T=300 \mathrm{~K}$. These larger systems required significantly longer runs to achieve a steady-state temperature profile. In particular, for $L_{z}=140.3 \mathrm{~nm}$, the total simulation time was $2.80 \mathrm{~ns}$, while for the $L_{z}=210.5 \mathrm{~nm}$ system the total simulation time was $6.72 \mathrm{~ns}$. We have estimated the simulation time required by computing the thermal diffusion length $l_{D}$ from the Einstein relation $l_{D}=\sqrt{6 D \tau}$, where $D$ is the thermal diffusivity and $\tau$ is the simulation time. From this expression, we estimate $l_{D}=170 \mathrm{~nm}$ for $\tau=6.72 \mathrm{~ns}$ and $\kappa$ $=1.5 \mathrm{~W} / \mathrm{mK}$, which is significantly larger than $L_{z} / 2$ when $L_{z}=210 \mathrm{~nm}$. Allowing for this long time required to reach steady state, the profiles were still averaged only over the last $0.56 \mathrm{~ns}$ of the simulation. While these results apparently show a systematic increase in $\kappa$ with increasing $L_{z}$, the effect is relatively small when compared to prior studies of the finite-size effects seen in pure systems including $\mathrm{Si}^{15}$ This is in agreement with our understanding of finite-size effects. ${ }^{15}$ In particular, finite-size effects are strong when the simulation cell length $L_{z}$ is comparable to the mean-free path of the vibrational modes. For the pure $\mathrm{Si}$ or Ge system, finite-size effects are substantial because the mean-free path can be quite large $(\sim 100 \mathrm{~nm}) .{ }^{15}$ By contrast, the mean-free path in the defective system is quite small. For example, at $x=0.10$ or $x=0.90$, the average spacing between point defects is comparable to the lattice parameter, and hence is in the range $\sim 0.5-0.6 \mathrm{~nm}$. As we will see in the next section, the simulation dramatically exaggerates the strength of point-defect scattering. While increasing $L_{z}$ might bring simulation into closer agreement with experiment, it is clear that the strong disagreement between experiment and simulation is not due to finite-size effects.

We did not explore finite-size effects for the directions perpendicular to the currents (i.e., the $x y$ plane). In our previous studies, we have found that for large enough simulation cells, the computed conductivity is fairly insensitive to system size. ${ }^{15}$

\section{THEORETICAL ANALYSIS}

We base the theoretical analysis on the approach applied by Abeles to $\mathrm{Si}-\mathrm{Ge}$ alloys. ${ }^{8}$ In this approach, the starting point is the high-temperature limit of the Callaway model. The elastic properties and mass density of the alloy are used to determine the resistivity $W_{p}$ of a defect-free virtual crystal. Point-defect scattering is then considered to further increase the resistivity $W_{p}$ of the virtual crystal.

The phenomenological model developed by Callaway ${ }^{16}$ is a useful approach to predict the thermal conductivity of bulk solids including alloys. The starting point for the model is the Boltzmann equation in the presence of a steady-state temperature gradient. Scattering rates for normal processes that conserve quasi-momentum are treated differently from the resistive processes including point-defect and Umklapp scattering. The thermal conductivity $\kappa$ in Callaway theory can be expressed as

$$
\kappa=\frac{\left(6 \pi^{2}\right)^{1 / 3}}{2 \pi^{2}} \frac{\left(k_{B} T\right)^{3}}{\hbar \Theta a}\left(I_{1}+\frac{I_{2}^{2}}{I_{3}}\right),
$$

where

$$
\begin{aligned}
& I_{1}=\int_{0}^{\Theta / T} \tau_{c} \frac{x^{4} e^{x}}{\left(e^{x}-1\right)^{2}} d x, \\
& I_{2}=\int_{0}^{\Theta / T} \frac{\tau_{c}}{\tau_{n}} \frac{x^{4} e^{x}}{\left(e^{x}-1\right)^{2}} d x, \\
& I_{3}=\int_{0}^{\Theta / T} \frac{\tau_{c}}{\tau_{n} \tau_{p}} \frac{x^{4} e^{x}}{\left(e^{x}-1\right)^{2}} d x,
\end{aligned}
$$

where $\Theta$ is the Debye temperature. From pertubation theory, the scattering rate $\tau_{p}^{-1}$ is given by

$$
\tau_{p}^{-1}=A \omega^{4}+B_{2} \omega^{2},
$$

where the first term is for point defects and the second term is for Umklapp scattering. The normal processes are described by the scattering rate $\tau_{n}^{-1}$ as

$$
\tau_{n}^{-1}=B_{1} \omega^{2} .
$$

The combined rate $\tau_{c}^{-1}$ is given by the usual expression

$$
\tau_{c}^{-1}=\tau_{n}^{-1}+\tau_{p}^{-1}
$$

The relative importance of normal and Umklapp scattering is captured by the parameter $\alpha=B_{1} / B_{2}$. For point-defect scattering, when a Debye model is assumed for the phonon spectra, the parameter $A=a^{3} / 4 \pi v^{3}$, where $a$ is the lattice parameter and $v$ is the phonon group velocity. In the Debye model dispersion is ignored, and the group velocity $v$ is independent on frequency or wavelength.

In the high-temperature limit where $T \gg \Theta$ the integrals can be performed. The simplification arises because in the high-temperature limit the phonon specific heat becomes $k_{B}$. The integrals have been evaluated by Abeles. ${ }^{8}$ The resulting expression for the thermal resistivity is 


$$
\begin{aligned}
\frac{W}{W_{p}}= & {\left[1+\left(\frac{5 \alpha}{9}\right)\right]\left[\frac{\tan ^{-1} U}{U}\right.} \\
& \left.+\frac{\left(1-\frac{\tan ^{-1} U}{U}\right)^{2}}{\left(\frac{1+\alpha}{5 \alpha}\right) U^{4}-\frac{1}{3} U^{2}+1-\frac{\tan ^{-1} U}{U}}\right]^{-1},
\end{aligned}
$$

where $W_{p}$ is the intrinsic resistivity of the virtual crystal without point-defect scattering,

$$
U^{2}=U_{0}^{2}\left(1+\frac{5 \alpha}{9}\right)^{-1},
$$

and

$$
U_{0}^{2}=\frac{(9 \pi / 2)^{1 / 3} \pi^{2} \hbar \Gamma a}{k_{B}^{2} \Theta W_{p}},
$$

where the scattering strength neglecting bond disorder is

$$
\Gamma=\sum_{i} x_{i}\left(\frac{M_{i}-M}{M}\right)^{2},
$$

where $x_{i}$ and $M_{i}$ are the fractional concentration and atomic mass of the component $i$ of the alloy. In the virtual crystal model, the alloy is treated as a perfect crystal with the phonons related to the elastic properties of the alloy. Therefore, the virtual crystal has the density and elastic properties of the alloy. Within this approach, we find the lattice parameter of the virtual crystal using Vergard's law,

$$
a=\sum_{i} x_{i} a_{i}
$$

and the atomic mass is given as

$$
M=\sum_{i} x_{i} M_{i}
$$

For the case of $\mathrm{Si}_{x} \mathrm{Ge}_{1-x}$, the relevant quantities simplify to

$$
\begin{aligned}
& \Gamma=x(1-x)\left(\frac{\Delta M}{M}\right)^{2}, \\
& \Delta M=M_{\mathrm{Ge}}-M_{\mathrm{Si}}, \\
& a=x a_{\mathrm{Si}}+(1-x) a_{\mathrm{Ge}}, \\
& M=x M_{\mathrm{Si}}+(1-x) M_{\mathrm{Ge}} .
\end{aligned}
$$

Abeles fit this model, including a term not shown above to account for bond disorder, to experimental data for a wide range of compositions. ${ }^{8}$ The fit resulted in prediction of $\alpha$ $=2.5$. It was found that the contribution of lattice strain to the scattering strength $\Gamma$ was only about $10 \%$. We show the model of Abeles for $T=300 \mathrm{~K}$ in Fig. 6. We also show in Fig. 6 the simulated data also shown in Fig. 5 using only the $\mathrm{Si}$ potential. We found that it was not possible to make a reasonable fit to the simulated data using the Abeles model described above. In particular, if we keep the value $\alpha=2.5$ fixed, we can only approximately fit the simulated data by increasing the value of $\Gamma$ by a factor of 25.8. This curve, which uses the experimental fit for $W_{p}$ found by Abeles, ${ }^{8}$ is also shown in Fig. 6. There is no particular justification to

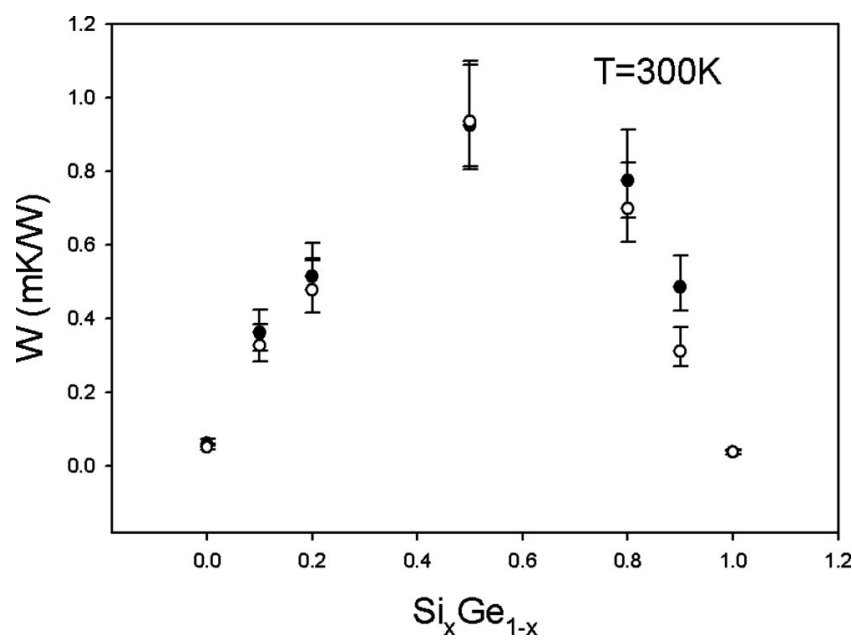

FIG. 5. Simulated resistivity $W$ for the $\mathrm{Si}$ (open circles) and Ge (closed circles) potentials. The alloy materials are simulated only with mass disorder. Comparison to Figs. 3 and 4 results show that mass disorder alone produces much of the thermal resistivity. The Ge potential results in systematically lower resistivity.

increase the value of $\Gamma$ in this way, and we include this curve only to illustrate the large discrepancies with existing experimental and theoretical results. Hence, we conclude that the point-defect scattering model used in the theory of Abeles based on the Debye spectrum is not adequate to explain the simulated data. We will return to discuss this point in the final section.

The simulations predict thermal resistivities greater than the theoretical results by a factor of between 4 and 6 for the alloys. More recent experimental results confirm this general trend. In particular, bulk and thin film alloys with composition $\mathrm{Si}_{0.8} \mathrm{Ge}_{0.2}$ have been measured to resistivity near $0.20 \mathrm{mK} / \mathrm{W} .{ }^{2,17}$ The thermal resistivity of $\mathrm{Si}_{0.84} \mathrm{Ge}_{0.16} / \mathrm{Si}_{0.74} \mathrm{Ge}_{0.26}$ superlattices was measured to be about $0.15 \mathrm{mK} / \mathrm{W}$, with very little observed interfacial

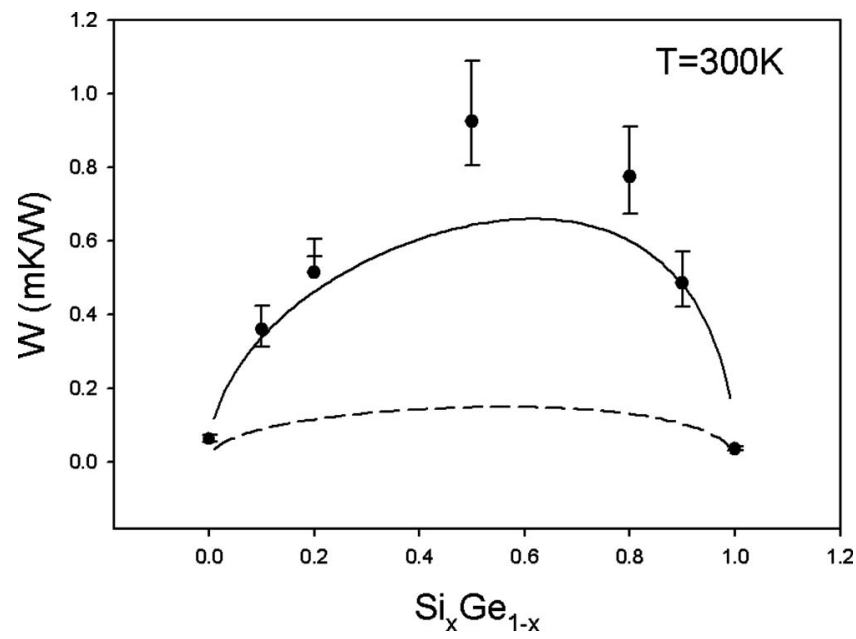

FIG. 6. The solid line shows the theoretical model fit to simulated data from Fig. 5 for the Si potential including only mass disorder. The dotted line shows the fit of Abeles to experimental data at $T=300 \mathrm{~K}$. It is apparent that the simulation predicts much larger resistivity $W$ than is observed in experiment. In order to approximately fit the simulation results to the theory, the strength of the alloy scattering was increased by a factor of 25.8 compared to the model used by Abeles to fit the experimental results. 
scattering. ${ }^{18}$ For $\mathrm{Si}_{0.9} \mathrm{Ge}_{0.1}$, values of about $0.10 \mathrm{mK} / \mathrm{W}$ have been found in another recent study. ${ }^{18}$ The variations between these different studies are likely due to differences in doping levels and grain size., ${ }^{2,17}$ However, each of the experimental results for the resistivity are consistently at least a factor of 4 smaller than our simulated values. Finally, in addition to being useful for predicting resistivity for alloys with high concentrations of point defects, it has been recently shown that the Abeles theory is adequate to predict resistivity in the limit of dilute alloy scattering. ${ }^{19}$

\section{DISCUSSION AND CONCLUSIONS}

We have computed the thermal resistivity $W$ of $\mathrm{Si}_{x} \mathrm{Ge}_{1-x}$ alloys using SW potentials. The resulting values of the resistivity $W$ are systematically too large by about a factor of 4-6, depending on concentration $x$. Fits to the simulated data using theoretical expressions based on the high-temperature limit of the Callaway model fail to adequately reproduce the simulation results. However, the theoretical analysis conclusively demonstrates that the simulation dramatically exaggerates scattering due to point defects. By comparing simulations where only mass disorder is present and bond disorder is eliminated, it is clear that the failure of the simulation model is not due to incorrect accounting of lattice strain.

Nevertheless, it is possible to speculate on the cause behind the dramatic failure of the model to predict the experimental results. The simulations here are entirely classical despite the fact that $T=300 \mathrm{~K}$ is significantly below the Debye temperature of $\mathrm{Si}(\Theta \approx 650 \mathrm{~K})$. The simulations at all temperatures will have a significant contribution from all phonon frequencies, which is not the case in the experiments. While for pure Si the comparison to experiment is reasonably good agreement near room temperature, this apparent success must arise somewhat accidentally. It is possible that introducing point defects highlights the failure of the classical model. Because point-defect scattering rate depends on frequency as $\omega^{4}$, high-frequency modes, including longitudinal and transverse optical modes, will be the most strongly scattered. In addition, it is expected that the predicted frequency dependence, which is determined using a Debye model, will break down at short wavelengths. Therefore, it is very likely that high-frequency modes excited in the classical simulation are very strongly scattered due to point defects, leading to unrealistically high resistivity $W$. By contrast, in the real system governed by quantum mechanics below the Debye temperature $\Theta$, many of these high-frequency modes are not excited.

In summary, we have highlighted a significant problem in using classical potentials to simulate thermal transport in alloys. For $\mathrm{Si}_{x} \mathrm{Ge}_{1-x}$ alloys, point-defect scattering results in unrealistically high resistivity $W$. While finite-size effects might be significant for our simulations, we predict that the simulated resistivity $W$ is about a factor of 4-6 larger than experimental results. This has important implications for any future studies of thermal transport in $\mathrm{Si}-\mathrm{Ge}$ materials, including, for example, alloys, superlattices, and nanowires. It may be that these results are fairly general and hence, might apply to simulations of other alloys and materials with large concentrations of point defects.

${ }^{1}$ H. Miyata, T. Yamada, and D. K. Ferry, Appl. Phys. Lett. 62, 2261 (1993). ${ }^{2}$ C. B. Vining, W. Laskow, R. R. Van der Beck, and P. D. Gorsuch, J. Appl. Phys. 69, 4333 (1991).

${ }^{3}$ C. B. Vining, J. Appl. Phys. 69, 331 (1991).

${ }^{4}$ A. Majumdar, Nature (London) 303, 777 (2004).

${ }^{5}$ D. Li, Y. Wu, R. Fan, P. Yang, and A. Majumdar, Appl. Phys. Lett. 83, 3186 (2003).

${ }^{6}$ W. Kim, J. Zide, A. Gossard, D. Klenov, S. Stremmer, A. Sharkouri, and A. Majumdar, Phys. Rev. Lett. 96, 045901 (2006).

${ }^{7}$ P. C. Weakliem and E. A. Carter, Phys. Rev. B 45, 13458 (1992).

${ }^{8}$ B. Abeles, Phys. Rev. 131, 1906 (1963).

${ }^{9}$ F. H. Stillinger and T. A. Weber, Phys. Rev. B 31, 5262 (1985).

${ }^{10}$ K. Ding and H. C. Anderson, Phys. Rev. B 34, 6987 (1986).

${ }^{11}$ M. Laradji, D. P. Landau, and B. Dunweg, Phys. Rev. B 51, 4894 (1995).

${ }^{12}$ M. Parrinello and A. Rahman, J. Appl. Phys. 52, 7182 (1981).

${ }^{13}$ J. Q. Broughton and X. Li, Phys. Rev. B 35, 9120 (1987).

${ }^{14}$ P. Jund and R. Jullien, Phys. Rev. B 59, 13707 (1999).

${ }^{15}$ P. K. Schelling, S. R. Phillpot, and P. Keblinski, Phys. Rev. B 65, 144306 (2002).

${ }^{16}$ J. Callaway, Phys. Rev. 113, 1046 (1959).

${ }^{17}$ S.-M. Lee, D. G. Cahill, R. Venkatasubramanian, and K. E. Goodson, Appl. Phys. Lett. 70, 2957 (1997).

${ }^{18}$ S. T. Huxtable, A. R. Abramson, C.-L. Tien, A. Majumdar, C. LaBounty, X. Fan, G. Zeng, and J. E. Bowers, Appl. Phys. Lett. 80, 1737 (2002).

${ }^{19}$ D. G. Cahill, F. Watanabe, and A. Rockett, Phys. Rev. B 71, 235202 (2005). 